\title{
Mean Square Relaxation Times for Evolution of Random Fields
}

\author{
Wayne G. Sullivan \\ Dublin Institute for Advanced Studies, Dublin, Ireland \\ Received April 1, 1974; in revised form July 30, 1974
}

\begin{abstract}
We consider the problem of relaxation times for Markov evolution of systems composed of a countable number of locally interacting particles, each one of which has a finite phase space. We give a theorem for comparison of mean square relaxation times of evolutions possessing the same ergodic stationary state. We give a reduction theorem for "attractive" evolutions. The results are applied to a generalization of the Glauber evolution of the one dimensional Ising chain.
\end{abstract}

\section{Introduction}

This paper concerns the stochastic time evolution of a system composed of a countable number of locally interacting particles, each one of which possessing only a finite number of states. A basic example of this type of system is the Glauber model [5] for the evolution of the one dimensional Ising chain.

The joint configuration of the particles is described by a point in the phase space $\Omega$. The equilibrium distribution is a probability measure $\mu$ on $\Omega$. We use the operator $T_{t}$ to express the evolution of the function $f$ on $\Omega$. The time development is specified by the master equation

$$
\frac{d}{d t}\left(T_{t} f\right)=G\left(T_{t} f\right)
$$

with a given master operator $G$.

The properties of the equilibrium state $\mu$ are usually well known. Very much less is known about the exact nature of the master operator. One fundamental constraint on $G$ is that it should have $\mu$ as a fixed point. A second commonly imposed constraint is that $G$ should satisfy the "principle of detailed balance". In probabilistic parlance this amounts to reversibility of $G$ with respect to $\mu$. The physical interpretation is that fluctuations from equilibrium should not distinguish the direction of time.

The particular concern of this paper is the convergence properties of solutions to (1.1) in the limit as $t$ approaches infinity. Assume there is a function space $\mathscr{F}$ which is complete in a certain norm $\|\cdot\|$ such that for each $f \in \mathscr{F}$ there is an $f_{\infty} \in \mathscr{F}$ with

$$
\lim _{t \rightarrow \infty} e^{\lambda t}\left\|T_{t} f-f_{\infty}\right\|=0
$$

for some fixed $\lambda \geqq 0$. Then $\lambda$ is called a relaxation coefficient for $T_{t}$ in the norm $\|\cdot\|$. The relaxation time of $T_{t}$ in the norm $\|\cdot\|$ is the reciprocal of the supremum of the set of relaxation coefficients. If there is a strictly positive relaxation coefficient, 
$T_{t}$ is said to have a finite relaxation time in the norm $\|\cdot\|$. If zero is the only relaxation coefficient, $T_{t}$ is called quasistable in the norm $\|\cdot\|$.

In considering models for the time development of the one dimensional Ising chain, Glauber [5] selected a certain $G$ which satisfied the two constraints mentioned above and had additional nice properties. For this $G$ he showed that one particle functions converge exponentially in time to their expectation values in the uniform norm $\|\cdot\|_{\infty}$,

$$
\|f\|_{\infty}=\sup _{x \in \Omega}|f(x)|
$$

Theorem 2 below then shows that any function of a finite number of particles has the same convergence property. A somewhat disturbing feature is that there are continuous functions on $\Omega$ which do not converge in this manner so that there are no nonzero relaxation coefficients for the Glauber evolution in $\|\cdot\|_{\infty}$.

Felderhof [4] represented the $G$ of the Glauber model as a symmetric operator and gave an explicit solution to the eigenvalue problem. The spectrum of $G$ consists of the eigenvalue zero corresponding to the equilibrium state and negative eigenvalues bounded away from zero corresponding to excited states. The gap in the spectrum implies that functions converge to their expectation values exponentially in time in the sense of (1.2) in the $\mu$-mean square norm. Theorem 1 below shows that a very broad class of master operators which leave $\mu$ fixed also converge in this manner, so that the finiteness of the $\mu$-mean square relaxation time is more a property of $\mu$ than of the specifics of the evolution. Mean square convergence is not sufficiently strong for some applications; nevertheless knowledge of mean square convergence properties gives information of a negative nature about convergence in stronger norms.

\section{Fundamentals}

The one particle phase space is the finite set $W$. The particles are labelled by the countable set $S$, which is taken to be $Z^{d}$, the set of points with integer coordinates in $d$-dimensional Euclidean space. The general phase space is $\Omega=W^{S}$. The discrete topology on $W$ makes $\Omega$ a compact Hausdorff space in the product topology. It is not difficult to generalize the results below to the case in which $S$ is an arbitrary countable set and each particle has its own compact metric space as phase space.

We consider several function spaces on $\Omega$. All functions are real valued unless otherwise specified. The symbol $\Lambda$ always denotes a finite subset of $S$. A function $g$ which has the property that the value of $g(x)$ depends only on the values of $x$ on $\Lambda$ is called a $\Lambda$-cylinder function. $C_{f}(\Omega)$ denotes the set of finite cylinder functions. Each $g \in C_{f}(\Omega)$ is a $\Lambda$-cylinder function for some $\Lambda$. $C(\Omega)$ denotes the space of continuous functions on $\Omega$ equipped with the norm $\|\cdot\|_{\infty}$ of (1.3).

Let $\mu$ be a probability measure on $\Omega$. We use the term measure to mean countable additive, bounded, Baire measure. The symbol $L^{p}(\mu), 1 \leqq p<\infty$, denotes the space of $p$-absolutely integrable functions on $\Omega$ equipped with the norm $\|\cdot\|_{p} . C_{f}(\Omega)$ is dense in $C(\Omega)$ and in $L^{p}(\mu), 1 \leqq p<\infty$, in the appropriate norms. 
The $\mu$-inner product is defined

$$
(f, g)_{\mu}=\int f g d \mu
$$

for $f, g \in C(\Omega)$. See Yosida [11] for details.

In working with the product space $\Omega$ some special notation is helpful. For specified $j \in S$, given $a \in W, x \in \Omega$, we employ the left subscript ${ }_{a} x$ to mean

$$
\left({ }_{a} x\right)_{k}=\left\{\begin{array}{lll}
a & \text { if } & k=j \\
x_{k} & \text { if } & k \neq j
\end{array} .\right.
$$

The symbol $j$ does not appear; it will be specified in the text or understood from context.

Definition 2.1. A generator $G$ on $\Omega$ is a formal sum $G=\Sigma G_{k}(a, b ; x)$ such that for each $k \in S, G_{k}(a, b ; x)$ is a continuous function on $W \times W \times \Omega$ which is independent of $x_{k}$ and satisfies

$$
\begin{gathered}
G_{k}(a, b ; x) \geqq 0 \quad \text { for } \quad a \neq b, \\
\sum_{b \in W} G_{k}(a, b ; x)=0
\end{gathered}
$$

for all $a, b \in W, x \in \Omega$. The operator of $G$ is the linear transformation with domain $C_{f}(\Omega)$ and values in $C(\Omega)$ whose action on the $\Lambda$-cylinder function $g$ is given by

$$
\begin{aligned}
G g & =\sum_{k \in \Lambda} G_{k} g, \\
G_{k} g\left({ }_{a} x\right) & =\sum_{b \in W} G_{k}(a, b ; x) g\left({ }_{b} x\right)
\end{aligned}
$$

with the modification of $x$ at the $k$-position.

Condition (2.4) makes the operator of $G$ map constant functions to zero. Also, by (2.4), it is sufficient to specify $G_{k}(a, b ; x)$ for $a \neq b$.

The above definition of a generator corresponds to the case in which only one particle at a time changes state. See Dobrushin [2] for generalization.

Definition 2.2. The generator $G$ on $\Omega$ is called reversible with respect to the probability measure $\mu$ if the operator of $G$ is symmetric in the $\mu$-inner product, i.e. if $(G f, g)_{\mu}=(f, G g)_{\mu}$ for all $f, g \in C_{f}(\Omega)$. $G$ is called strictly $\mu$-reversible if each $G_{k}$ is $\mu$-reversible.

When the probability measure $\mu$ on $\Omega$ is the equilibrium state of a "reasonable" potential, it possesses certain well-behaved conditional probabilities and vice-versa (see Sullivan [9]). We assume that $\mu$ possesses a family of strictly positive functions $\left\{\mu_{k}(a \mid x): k \in S\right\}$ such that $\mu_{k}(a \mid x)$ is independent of $x_{k}$ and for all $k \in S, a \in W$, $x \in \Omega, f \in C(\Omega)$

$$
\int\left[\sum_{a \in W} f\left({ }_{a} x\right) \mu_{k}(a \mid x)\right] \mu(d x)=\int f d \mu
$$

with the modification of $x$ at the $k$-position.

For such $\mu$ there are several associated generators. Two examples are the type $I$ generator, $G^{I}=\Sigma G_{k}^{I}$, and the type $I I$ generator, $G^{I I}=\Sigma G_{k}^{I I}$, of $\mu$ given by

$$
\begin{aligned}
G_{k}^{I}(a, b ; x) & =\mu_{k}(b \mid x), & & b \neq a, \\
G_{k}^{I I}(a, b ; x) & =\left[\mu_{k}(b \mid x) / \mu_{k}(a \mid x)\right]^{\frac{1}{2}}, & & b \neq a .
\end{aligned}
$$

Both $G^{I}$ and $G^{I I}$ are strictly reversible with respect to $\mu$. 
The difficulty in solving the master Eq. (1.1) is due to the the fact that, in general, a generator $G$ on $\Omega$ is an unbounded operator. We approach the problem from the point of view of semigroup theory using the results of Liggett [7] and Sullivan [10]. The reader is referred to Yosida [11] as a basic reference on semigroups. We use the term "strongly continuous" to mean "of class $C_{0}$ " in Yosida's terminology. An operator $T$ on a space $\mathscr{F}$ with norm $\|\cdot\|$ is called a contraction if $\|T f\| \leqq\|f\|$ for all $f \in \mathscr{F}$. An operator $G$ defined on a domain in $\mathscr{F}$ is called dissipative if for each $f$ in the domain of $G$ and each $\lambda>0$

$$
\|f-\lambda G f\| \geqq\|f\| \text {. }
$$

In a real inner product space with $\|f\|=(f, f)^{\frac{1}{2}}$ this amounts to the condition

$$
(G f, f) \leqq 0
$$

for each $f$ in the domain of $G$. The closure $\bar{G}$ of $G$ is defined so that the graph of $\bar{G}$ is the closure of the graph of $G$. This depends on the norm but we use the one symbol $\bar{G}$ with the norm to be taken from context.

Let $G=\Sigma G_{k}$ be a generator on $\Omega$. The operator $\sum_{k \in A} G_{k}$ is a bounded linear dissipative operator on $C(\Omega)$ and we can evaluate the associated evolution $\exp \left(t \sum_{k \in \Lambda} G_{k}\right), t \geqq 0$, by the exponential power series. In many cases of interest these evolutions converge with increasing $\Lambda$ to a well-defined evolution $T_{t}$, $t \geqq 0$, corresponding to $G$ (see $[7,10]$ ).

Definition 2.3. The generator $G=\Sigma G_{k}$ on $\Omega$ is said to have the semigroup property if the closure $\bar{G}$ of $G$ as defined on $C_{f}(\Omega)$ in $C(\Omega)$ is the infinitesimal generator of a strongly continuous, linear semigroup of contractions $T_{t}, t \geqq 0$, such that for each $t_{0}>0$ and each $f \in C(\Omega)$

$$
\lim _{\Lambda \rightarrow S} \sup _{0 \leqq t \leqq t_{0}}\left\|T_{t} f-\exp t \sum_{k \in \Lambda} G_{k} f\right\|_{\infty}=0
$$

where the $\Lambda$-limit is taken in the sense of the net of finite subsets of $S$ ordered by inclusion.

When $G$ has the semigroup property with evolution $T_{t}$ acting on $C(\Omega)$, we use topological vector space duality to get the associated evolution $T_{t}^{\prime}$ acting on measures (see Yosida [11]). A probability measure $\mu$ is called stationary with respect to $G$ (or under $T_{t}$ ) if $T_{t}^{\prime} \mu=\mu$ for all $t \geqq 0$. By differentiation one obtains the following.

Lemma 2.4. Let the generator $G$ on $\Omega$ have the semigroup property. A necessary and sufficient condition that the probability measure $\mu$ be stationary with respect to $G$ is that $\int G f d \mu=0$ for all $f \in C_{f}(\Omega)$.

Proposition 2.5. Let the generator $G$ on $\Omega$ have the semigroup property and be reversible with respect to the probability measure $\mu$. Then $\mu$ is stationary with respect to $G$.

\section{Ergodicity and a Comparison Theorem}

From Yosida [11], XIII. 1, it is easy to deduce the following two propositions.

Proposition 3.1. Let the generator $G$ on $\Omega$ have the semigroup property with evolution $T_{t}$. Let $\mu$ be a probability measure which is stationary with respect to $G$. 
Then the action of $T_{t}$ on $C(\Omega)$ extends uniquely to a strongly continuous linear semigroup of contractions on $L^{p}(\mu)$ for $1 \leqq p<\infty$. The associated infinitesimal generator of the semigroup is the closure in the p-norm of $G$ as defined on $C_{f}(\Omega)$ and is a closed linear dissipative operator in $L^{p}(\mu)$. We have

$$
(G f, f)_{\mu} \leqq 0
$$

for each $f \in C_{f}(\Omega)$.

Proposition 3.2. Let $G$ and $\mu$ satisfy the hypothesis of Proposition 3.1 and further assume that $\mu$ is reversible with respect to $G$. Let $\bar{G}$ denote the $L^{2}(\mu)$ closure of $G$. Then $\bar{G}$ is self-adjoint and its spectrum consists of nonpositive real numbers. Let $-\lambda$ be the supremum of the strictly negative spectrum of $\bar{G}$ and let $P_{0}$ denote the projection on the zero eigenspace of $\bar{G}$. Then for each $f \in L^{2}(\mu), t \geqq 0$

$$
\begin{gathered}
\lim _{t \rightarrow \infty}\left\|T_{t} f-P_{0} f\right\|_{2}=0, \\
\left\|e^{\lambda t}\left(T_{t} f-P_{0} f\right)\right\|_{2} \leqq\left\|f-P_{0} f\right\|_{2} .
\end{gathered}
$$

It follows that, for the evolution of Proposition 3.2, if $\lambda>0$, then any $\lambda^{\prime}$, $0 \leqq \lambda^{\prime}<\lambda$, is a relaxation coefficient and the $L^{2}(\mu)$ relaxation time is $1 / \lambda$.

When one has a Markov process on a finite set, the set can be partitioned into a transient class and a number of ergodic classes (see Doob [3]). Within each ergodic class the process proceeds independently of the configuration of the other ergodic classes. Each ergodic class has a characteristic relaxation time. A similar decomposition for locally compact spaces can be found in Yosida [11], XIII.4.

Definition 3.3. The evolution $T_{t}$ is called ergodic it it possesses a unique stationary probability measure. If $T_{t}$ possesses a stationary probability measure $\mu$ such that the only fixed points of $T_{t}$ in $L^{2}(\mu)$ are the constant functions, then $T_{t}$ is called $L^{2}(\mu)$ ergodic.

It is not difficult to show the following:

Proposition 3.4. Let the generator $G$ on $\Omega$ have the semigroup property with evolution $T_{t}$. If $T_{t}$ is ergodic with stationary probability measure $\mu$, then $T_{t}$ is $L^{2}(\mu)$ ergodic.

Theorem 1. Let $G$ and $H$ be generators on $\Omega$ which have the semigroup property with corresponding evolutions $T_{t}$ and $U_{t}$. Let $G$ be reversible with respect to the probability measure $\mu$ and let $T_{t}$ be $L^{2}(\mu)$ ergodic. Assume

$$
(H f, f)_{\mu} \leqq(G f, f)_{\mu}
$$

for all $f \in C_{f}(\Omega)$. Then $\mu$ is stationary with respect to $H$ and $U_{t}$ is $L^{2}(\mu)$ ergodic. If $\lambda \geqq 0$ is such that

$$
e^{\lambda t}\left\|T_{t} f-\int f d \mu\right\|_{2}
$$

is bounded in $t \geqq 0$ for each $f \in L^{2}(\mu)$, then

$$
e^{\lambda t}\left\|U_{t} f-\int f d \mu\right\|_{2} \leqq\left\|f-\int f d \mu\right\|_{2}
$$

Proof. From (3.4) we deduce that $H$ is dissipative with respect to $(\cdot, \cdot)_{\mu}$. It follows easily that $\mu$ is stationary under $U_{t}$. Let $f \in L^{2}(\mu)$ be a fixed point of $U_{t}$. 
Then $\bar{H} f=0$. From (3.4) and reversibility we conclude that $f$ is in the domain of $(-\bar{G})^{\frac{1}{2}}$ and $(-\bar{G})^{\frac{1}{2}} f=\bar{G} f=0$. Hence $f$ is a fixed point of $T_{t}$, so $U_{t}$ is $L^{2}(\mu)$ ergodic. Let $L_{0}^{2}(\mu)$ denote the subspace of $L^{2}(\mu)$ consisting of those functions whose $\mu$ integral vanishes. From the boundedness of (3.5) using the spectral representation we find that the restriction of $G+\lambda$ to $C_{f}(\Omega) \cap L_{0}^{2}(\mu)$ is dissipative. From (3.4) we have that the restriction of $H+\lambda$ to $C_{f}(\Omega) \cap L_{0}^{2}(\mu)$ is dissipative, so $e^{\lambda t} U_{t}$ is a contraction semigroup on $L_{0}^{2}(\mu)$ which yields (3.6).

Corollary 3.5. The $L^{2}(\mu)$ relaxation time of $U_{t}$ is no greater than that of $T_{t}$.

An important case in which (3.4) is satisfied is when $G$ and $H$ satisfy the remaining hypotheses of Theorem 1 and $H=G+K$ where $K$ is a generator on $\Omega$ which is either strictly reversible with respect to $\mu$ or has the semigroup property with stationary measure $\mu$.

\section{Attractive Evolutions}

In the case of "attractive" evolutions one can reduce many convergence questions to considerations involving one particle functions. The term "attractive" is from Holley [6]. Theorem 2 below generalizes his result. See Preston [8] for fundamentals of the techniques used here.

We assume that the one particle phase space is equipped with a partial order $\leqq$ which has a minimal element 0 and a maximal element $w$ so that each $a \in W$ satisfies $0 \leqq a \leqq w$. The general phase space $\Omega$ has the product partial order so that $x \leqq y$ means $x_{k} \leqq y_{k}$ for all $k \in S$. In this order $\Omega$ has unique maximal element, denoted $w$, and unique minimal element, denoted 0 , so that for each $x \in \Omega, 0 \leqq x \leqq w$. One determines from context whether $0, w$ refer to points of $W$ or $\Omega$.

Definition 4.1. The space of continuous nondecreasing functions on $\Omega$ is denoted $C_{i}(\Omega)$. A generator $G=\Sigma G_{k}$ on $\Omega$ is called attractive if for each $k \in S$, there exists a $\tau_{k}>0$ such that $\left(1+\tau_{k} G_{k}\right)$ maps $C_{i}(\Omega)$ into itself.

Proposition 4.2. Let the attractive generator $G$ on $\Omega$ have the semigroup property with evolution $T_{q}$. Then $T_{t}, t \geqq 0$, maps $C_{i}(\Omega)$ into itself.

Let $\mu$ be a "well-behaved" probability measure which is attractive in the sense of Preston [8]. Then the type $I$ generator of $\mu$ given by (2.6) is attractive. Other generators associated with $\mu$ are not necessarily attractive.

Lemma 4.3. Let $G$ be an attractive generator on $\Omega$ which has the semigroup property with evolution $T_{t}$. Let $f, g \in C_{i}(\Omega)$ be such that for some $\lambda \geqq 0$,

$$
\begin{aligned}
\lim _{t \rightarrow \infty} e^{\lambda t}\left[T_{t} f(w)-T_{t} f(0)\right] & =\lim _{t \rightarrow \infty} e^{\lambda t}\left[T_{t} g(w)-T_{t} g(0)\right]=0 .
\end{aligned}
$$

Then

$$
\lim _{t \rightarrow \infty} e^{\lambda t}\left\|T_{t}(f g)\right\|_{\infty}=0 .
$$

Proof. Since we can add constants and multiply by positive factors, there is no loss in generality in assuming that the values of $f$ and $g$ lie in $[0,1]$. Then $f g$ and $f+g-f g$ are nondecreasing so

$$
0 \leqq T_{t}(f g)(w)-T_{t}(f g)(0) \leqq T_{t}(f+g)(w)-T_{t}(f+g)(0) .
$$


Theorem 2. Let $G$ be an attractive generator on $\Omega$ which has the semigroup property. Let $\mathscr{F}$ be a set of nondecreasing functions in $C(\Omega)$ such that the algebra generated by $\mathscr{F}$ is dense in $C(\Omega)$ in the norm $\|\cdot\|_{\infty}$. If there exists a $\lambda \geqq 0$ such that

$$
\lim _{t \rightarrow \infty} e^{\lambda t}\left[T_{t} f(w)-T_{t} f(0)\right]=0
$$

for each $f \in \mathscr{F}$, then $T_{t}$ has a unique stationary probability measure $\mu$ and for each $g$ in the algebra generated by $\mathscr{F}$

$$
\lim _{t \rightarrow \infty} e^{\lambda t}\left\|T_{t} g-\int g d \mu\right\|_{\infty}=0
$$

Also, for each $f \in C(\Omega)$

$$
\lim _{t \rightarrow \infty}\left\|T_{t} f-\int f d \mu\right\|_{\infty}=0 .
$$

If $G$ is $\mu$-reversible, then $\lambda$ is an $L^{2}(\mu)$ relaxation coefficient.

Proof. For $g$ in the algebra generated by $\mathscr{F}$ we define $\mu[g]$ by

$$
\mu[g]=\lim _{t \rightarrow \infty} T_{t} g(w)
$$

which exists for any nondecreasing function, hence for any finite linear combination of nondecreasing functions. Limit (4.4) defines a continuous linear functional on a dense subspace of $C(\Omega)$, so there is a measure on $\Omega$ also denoted $\mu$ such that

$$
\mu[g]=\int g d \mu
$$

for each $g$ in the algebra generated by $\mathscr{F}$. The properties of $T_{t}$ imply that $\mu$ is a probability measure. Lemma 4.3 then gives (4.2). The uniqueness of $\mu$ and limit (4.3) follow easily. The assertion that $\lambda$ is an $L^{2}(\mu)$ relaxation coefficient follows from the spectral representation of the closure of $G$.

Usually $\mathscr{F}$ is a set of one particle functions which generates the algebra $C_{f}(\Omega)$. Convergence factors other than exponentials could be used in Theorem 2 .

\section{A Generalized Glauber Model}

The Glauber evolution [5] with variable coupling serves well to illustrate the preceding results. We consider the one dimensional Ising chain with variable nearest neighbour interactions in zero magnetic field. The phase space is $\Omega=\{-1,1\}^{Z}$. In the configuration $x \in \Omega$, the interaction energy between sites $k$ and $k+1$ is $-J_{k} x_{k} x_{k+1}$. We consider the generator $G=\Sigma G_{k}$ with

$$
\begin{aligned}
G_{k}(a, b ; x) & =-\frac{1}{2} a b\left(1-\alpha_{k} a x_{k-1}-\beta_{k} a x_{k+1}\right), \\
\alpha_{k} & =\frac{1}{2}\left[\tanh \left(J_{k-1}+J_{k}\right)+\tanh \left(J_{k-1}-J_{k}\right)\right], \\
\beta_{k} & =\frac{1}{2}\left[\tanh \left(J_{k-1}+J_{k}\right)-\tanh \left(J_{k-1}-J_{k}\right)\right],
\end{aligned}
$$

for $a, b \in\{-1,+1\}$. From results in [7] it follows that $G$ has the semigroup property. If $v$ is a probability measure on $\Omega$ whose one point conditional probabilities satisfy

$$
v_{k}(a \mid x)=\exp \left(J_{k-1} x_{k-1} a+J_{k} x_{k+1} a\right) / Z_{k x}
$$


for each $k \in Z, a \in\{-1,+1\}, x \in \Omega$, where $Z_{k x}$ is chosen so that the sum of $v_{k}(a \mid x)$ over the two values of a is one, then $G$ is strictly reversible with respect to $v$.

One stationary probability measure of the Glauber generator $G$ of (5.1) is the inhomogeneous Markov chain $\mu$ such that

$$
\begin{aligned}
\mu\left(x_{k}=a\right) & =\frac{1}{2}, \\
\mu\left(x_{k+1} \mid x_{k}\right) & =\frac{1}{2}\left(1+x_{k} x_{k+1} \tanh J_{k}\right) .
\end{aligned}
$$

We shall show below that $\mu$ is the only stationary probability measure of $G$ when the coupling constants are bounded. The type $I$ generator (2.6) of $\mu$ coincides with the Glauber generator (5.1).

Now we consider the very special case when all the $J_{k}$ 's are zero. Any concept of relaxation time which fails for this case seems doomed for more complex evolutions. One can compute the evolution of point measures to get the following:

Lemma 5.1. Consider the Glauber generator (5.1) with all the $J_{k}$ 's zero. Let

$$
f_{n}(x)=\operatorname{sgn}\left(\sum_{k=1}^{n} x_{k}\right)
$$

where $\operatorname{sgn}(u)=+1,0$ or -1 as $u$ is positive, zero or negative. Then for each fixed $t>0$

$$
\begin{aligned}
& \lim _{n \rightarrow \infty} T_{t} f_{n}(+)=1, \\
& \lim _{n \rightarrow \infty} T_{t} f_{n}(-)=-1,
\end{aligned}
$$

where " + "indicates the all +1 point of $\Omega$ and " - " the all -1 point.

Proposition 5.2. If $\lambda>0$, then $\lambda$ is not a relaxation coefficient for the Glauber evolution with all $J_{k}$ 's zero in the $\|\cdot\|_{\infty}$ norm on $C(\Omega)$.

We return to the general case. There is no loss in generality in assuming that $J_{k} \geqq 0$ for all $k$, as we can relabel the direction of individual spins to achieve this. One verifies the Glauber evolution with $J_{k} \geqq 0$ for all $k$ is attractive in the ordering of $\Omega$ induced from the $-1<1$ ordering on $\{-1,1\}$.

Let $s_{k}$ denote the element of $C(\Omega)$ such that $s_{k}(x)=x_{k}$. Each element $v \in \ell^{1}(Z)$ yields an element of $C(\Omega)$ under the correspondence

$$
v \mapsto \sum_{k \in Z} v_{k} s_{k} .
$$

This mapping is norm preserving and the image of $\ell^{1}(Z)$ in $C(\Omega)$ is a closed linear subspace which we denote by $\mathscr{F}$. The operator $G$ of $(5.1)$ maps $\mathscr{F}$ into itself and yields the following differential equation for $v(t) \in \ell^{1}(Z)$ via (5.4):

$$
\begin{aligned}
\frac{d}{d t} v(t) & =B v(t) \\
{[B v]_{k} } & =\beta_{k-1} v_{k-1}-v_{k}+\alpha_{k+1} v_{k+1} .
\end{aligned}
$$

One verifies that $B$ is a bounded dissipative operator on $\ell^{1}(Z)$, so that $\exp (t B)$, $t \geqq 0$, is a contraction semigroup. We deduce that $\mathscr{F}$ is invariant under $T_{t}$ and that $T_{t}$ agrees with $\exp (t B)$ in $\mathscr{F}$ through the correspondence (5.4). 
If the $J_{k}$ 's are uniformly bounded,

$$
\left|J_{k}\right| \leqq J \quad \text { all } \quad k \in Z
$$

let $\lambda$ satisfy $0 \leqq \lambda \leqq 1-\tanh 2 J$. We deduce that $B+\lambda$ is dissipative in $\ell^{1}(Z)$. Hence $\exp [t(B+\lambda)]$ is a contraction semigroup on $\mathscr{F}$ and

$$
\left\|e^{\lambda t} T_{t} \sum_{k \in Z} v_{k} s_{k}\right\|_{\infty} \leqq \sum_{k \in Z}\left|v_{k}\right|
$$

From (5.7) and Theorem 1 and 2 we have the following:

Proposition 5.3. For the Glauber generator $G$ of (5.1) which satisfies (5.6) there is a unique stationary probability measure $\mu$ which is the inhomogeneous Markov chain satisfying (5.3). For each $g \in C_{f}(\Omega)$ and each $\lambda$ satisfying $0 \leqq \lambda<1-\tanh 2 J$ we have

$$
\lim _{t \rightarrow \infty} e^{\lambda t}\left\|T_{t} g-\int g d \mu\right\|_{\infty}=0 .
$$

The $L^{2}(\mu)$ relaxation time is no greater than $1 /(1-\tanh 2 J)$. The type II generator of $\mu$ has a finite $L^{2}(\mu)$ relaxation time.

Next let us consider the case where the $J_{k}$ 's may be unbounded but strictly positive. Define $u_{k}$ by

$$
\exp \left(-u_{k}\right)=\tanh J_{k}
$$

Next define the probability measure $\mu_{+}$by taking limits of finite chains of spins with one point conditional probabilities satisfying (5.2) subject to boundary spins equal to +1 . Define $\mu_{-}$similarly with boundary condition -1 . If $\Sigma u_{k}<\infty$, then $\mu_{+} \neq \mu_{-}$. This is a phase transition in the sense of Dobrushin [1]. The probability measure $\frac{1}{2} \mu_{+}+\frac{1}{2} \mu_{-}$is not a Markov chain even though it satisfies (5.2). This kind of phase transition is not a singularity of a partition function. Instead it is the existence of more than one equilibrium state obtainable from the potential by taking limits of finite systems with different boundary conditions. In the translation invariant case one needs long range forces to obtain a one dimensional phase transition. Here we have nearest neighbour forces which are not translation invariant nor bounded.

Another interesting case is that in which $\lim u_{k}=0, \sum_{k>0} u_{k}=\infty$ and $\sum_{k<0} u_{k}=\infty$. Here it can be shown that the only stationary probability measure for $G$ is the $\mu$ of (5.3). By considering the action of $G$ on one particle functions one can show that zero is a limit point of the spectrum of $G$ considered as an operator on $L^{2}(\mu)$.

Proposition 5.4. Let $G$ be the Glauber generator (5.1) with strictly positive $J_{k}$ 's related to $u_{k}$ 's by (5.8). If $\Sigma u_{k}<\infty$, there is a phase transition in the sense of there being more than one reversible stationary probability measure for $G$. If $\lim u_{k}=0$, $\sum_{k>0} u_{k}=\infty$ and $\sum_{k<0} u_{k}=\infty$, then the only stationary probability measure for $G$ is the inhomogeneous Markov chain $\mu$ of (5.3), but the evolution is quasistable in the sense that the only $L^{2}(\mu)$ relaxation coefficient is zero. 
For higher dimensional, translation invariant Ising models which have only one phase, i.e. above the critical temperature or in a nonzero magnetic field, it would be interesting to know if $L^{2}$ quasistability can occur with "reasonable" master operators.

I wish to acknowledge valuable discussions with Professor J. T. Lewis.

\section{References}

1. Dobrushin, R. L.: Theory Probability Appl. 13, 197-224 (1968)

2. Dobrushin, R. L.: Problems of Information Transmission 7, 149-64; 7, 235-241 (1971)

3. Doob, J. L.: Stochastic Processes. New York: John Wiley \& Sons 1953

4. Felderhof, B. U.: Rep. Math. Phys. 1, 215-234 (1970); 2, 151-152 (1971)

5. Glauber, R. J.: J. Math. Phys. 4, 294-207 (1963)

6. Holley, R.: Z. Wahrscheinlichkeitstheorie verw. Geb. 24, 325-334 (1972)

7. Liggett, T. M.: Trans. Am. Math. Soc. 165, $471-481$ (1972)

8. Preston, C. J.: Specification of random fields I and II. To appear.

9. Sullivan, W. G.: Commun. math. Phys. 33, 61-74 (1973)

10. Sullivan, W.G.: A unified existence and ergodic theorem for Markov evolution of random fields. To appear

11. Yosida, K.: Functional Analysis. Berlin-Heidelberg-New York: Springer 1965

Communicated by J. L. Lebowitz
Wayne G. Sullivan

Dublin Institute for Advanced Studies

10 Burlington Road

Dublin 4, Ireland 